\title{
THE IMBEDDING OPERATORS ON A PARTIALLY ORDERED SET
}

\author{
G. B. ROBISON AND E. S. WOLK
}

1. Introduction. Let $P$ be any partially ordered set, with a greatest element $I$ and a least element $0.2^{P}$ will denote the set of all subsets of $P$. We denote by $\mathscr{G}(P)$ the collection of set operators on $P$ (i.e., functions on $2^{P}$ into $2^{P}$ ) with the following properties for any subsets $S$ and $T$ contained in $P$ :

(i) $S \subseteq \phi(S)$ (extensive property),

(ii) if $S \subseteq T$, then $\phi(S) \subseteq \phi(T)$ (monotonic property),

(iii) $\phi(\phi(S))=\phi(S)$ (idempotent property),

(iv) if $S$ consists of a single point $p$, then $\phi(S)=\phi(p)=\{x \in P \mid x \leqq p\}$ (imbedding property).

(We denote set inclusion by $\subseteq$, reserving $\subset$ for proper inclusion.) Operators satisfying (i), (ii), and (iii) are called closure operators (Birkhoff [1]). Following Ward [3] and Dilworth and McLaughlin [2], closure operators with property (iv) will be called imbedding operators.

In any collection of set operators on $P$, a partial ordering may be defined by:

$$
\phi_{1} \leqq \phi_{2} \text { if and only if } \phi_{1}(S) \subseteq \phi_{2}(S) \text { for every } S \in 2^{P} .
$$

If $\left\{\phi_{t}\right\}$ is any collection of imbedding operators, we may define a g.l.b. and l.u.b. of $\left\{\phi_{t}\right\}$ by:

$$
\begin{aligned}
& {\left[\wedge\left\{\phi_{t}\right\}\right](S)=\cap\left\{\phi_{t}(S)\right\},} \\
& {\left[\bigvee\left\{\phi_{t}\right\}\right](S)=\cap\left\{\psi(S) \mid \psi \in g(P) \text { and } \psi \geqq \phi_{t} \text { for all } t_{\}} .\right.}
\end{aligned}
$$

Under these definitions $g(P)$ is a complete lattice $[2 ; 3]$.

Dilworth and McLaughlin have found the concept of imbedding operator a useful tool in their investigation of distributivity in lattices [2], and Ward [3] has emphasized its significance for imbedding problems. However, no attention has yet been given to the structure of the lattice $g(P)$ itself. It is the purpose of this paper to investigate some of the properties of $g(P)$ which appear to be of interest. We obtain some general theorems concerning the structure of $g(P)$, as well as some results of independent interest relating to imbedding operators. We also obtain certain conditions on $P$ which are sufficient for $g(P)$ to be a chain.

Received by the editors July 13, 1956. 
2. Preliminaries. If $\phi$ and $\psi$ are set operators on $P, \phi \psi$ is the operator defined by $[\phi \psi](S)=\phi(\psi(S))$. For convenience of later reference we state the following easily proved properties of closure operators.

Lemma A. Let $\phi$ and $\psi$ be any set operators on $P$.

(1) If $\phi$ satisfies (i) and $\psi$ satisfies (ii) and (iii), then $\phi \leqq \psi$ implies $\phi \psi=\psi \phi=\psi$.

(2) If $\psi$ satisfies (i), then $\psi \phi=\psi$ implies $\phi \leqq \psi$.

(3) If $\psi$ satisfies (i) and $\phi$ satisfies (ii), then $\phi \psi=\psi$ implies $\phi \leqq \psi$.

$S$ is a $\phi$-closed set or a $\phi$-ideal if $\phi(S)=S$. If $S=\phi(p)$ for some element $p \in P, S$ is called a principal ideal.

We now define two operators on $P$ as follows:

$$
\begin{aligned}
& S^{*}=\{x \in P \mid x \geqq y \text { for all } y \in S\}, \\
& S^{+}=\{x \in P \mid x \leqq y \text { for all } y \in S\} .
\end{aligned}
$$

Note that if $S \subseteq T$, then $S^{*} \supseteq T^{*}$ and $S^{+} \supseteq T^{+}$. Two operators which play special roles as least and greatest elements of $\mathscr{g}(P)$ respectively can then be defined by

$$
\begin{aligned}
& \omega(S)=\{x \in P \mid x \leqq y \text { for some } y \in S\}, \\
& \nu(S)=\left(S^{*}\right)^{+} .
\end{aligned}
$$

Note that with this definition (iv) can be restated as $\phi(p)=\omega(p)$.

From the definition it is clear that

Lemma B. For any collection $\left\{S_{t}\right\}$ of subsets of $P, \cup\left\{\omega\left(S_{t}\right)\right\}$ $=\omega\left(\bigcup\left\{S_{t}\right\}\right)$.

For the sake of completeness, we include a proof of the following known result.

THEOREM 1. If $\phi \in \mathscr{I}(P)$, then $\omega \leqq \phi \leqq \nu$.

Proof. If $x \in \omega(S)$, there exists $y \in S$ with $x \leqq y$. By (iv) and (ii), $x \in \phi(y) \subseteq \phi(S)$, and so $\omega \leqq \phi$. If $q \in S^{*}$, then $S \subseteq \omega(q)=\phi(q)$. By (ii) and (iii), $\phi(S) \subseteq \phi(q)$; so $q \in(\phi(S))^{*}$. Since $q$ was any element of $S^{*}$, $S^{*} \subseteq(\phi(S))^{*}$, and $\nu(S)=S^{*+} \supseteq(\phi(S))^{*+}=\nu(\phi(S)) \supseteq \nu(S)$ by (i) and (ii). Hence $\nu \phi=\nu$, and so by Lemma A, $\phi \leqq \nu$.

Definition. If $\Sigma \subseteq 2^{P}$, then $\Sigma$ is a generating collection if and only if

(1) each element of $\Sigma$ is $\omega$-closed,

(2) $\Sigma$ is closed under set intersection,

(3) $\Sigma$ contains all principal ideals of $P$.

For each $\phi$, let us denote by $\Sigma(\phi)$ the collection of all $\phi$-ideals. The following properties are easily established: 
LeMma C. (1) $\phi_{1}=\phi_{2}$ if and only if $\Sigma\left(\phi_{1}\right)=\Sigma\left(\phi_{2}\right)$.

(2) $\Sigma(\phi)$ is a generating collection.

For any generating collection $\Sigma$, let us denote by $\phi_{\Sigma}$ the operator defined by $\phi_{\Sigma}(S)=\bigcap\{A \in \Sigma \mid S \subseteq A\}$. We note that the intersection closure property of $\Sigma$ insures that $\phi_{\Sigma}(S) \in \Sigma$ for any $S \in 2^{P}$. The following results may then be easily verified:

Lemma D. (1) For any generating collection $\Sigma, \phi_{\Sigma} \in \mathfrak{g}(P)$ and $\Sigma\left(\phi_{\Sigma}\right)=\Sigma$.

(2) For any $\phi \in \mathscr{I}(P), \phi_{\Sigma(\phi)}=\phi$.

(3) If the generating collections are partially ordered by inclusion, the correspondence $\phi \rightarrow \Sigma(\phi)$ is a 1:1 order-inverting mapping of $g(P)$ onto the set of all generating collections of $P$.

Part (1) of this lemma gives us an imbedding operator whenever we have a generating collection. The following lemma describes another method for constructing certain imbedding operators.

Lemma E. For a pair of fixed sets $A$ and $B$ contained in $P$, let a set operator $\psi$ be defined as follows:

$$
\begin{aligned}
\psi(S) & =\omega(S) \text { if } A \nsubseteq \omega(S), \\
& =\omega(S) \cup \omega(B) \text { if } A \subseteq \omega(S) .
\end{aligned}
$$

Then $\psi \in g(P)$ if and only if $B \subseteq \nu(A)$.

We omit the proof.

If we denote an imbedding operator of the above kind by $\left.{ }_{\mathbf{A}}^{B}\right]$, then the following results may be established:

Corollary 1. (1) $A_{1} \subseteq A_{2}$ implies $\left.\left.{ }_{A_{1}}^{B}\right] \geqq{ }_{A_{2}}^{B}\right]$ and

(2) $B_{1} \subseteq B_{2}$ implies $\left.\left.{ }_{A}^{B_{1}}\right] \leqq{ }_{A}^{B_{2}}\right]$.

Corollary 2. If $\left\{B_{t}\right\}$ is a collection of subsets of $\nu(A)$, then the operator defined by $\left.\psi(S)=\bigcup\left\{\begin{array}{l}B_{t} \\ A\end{array}\right](S)\right\}$ is an imbedding operator. Similarly, if $B \subseteq \nu\left(A_{t}\right)$ for each $A_{t}$ in a collection $\left\{A_{t}\right\}$, then $\left.\phi(S)=\bigcup\left\{\begin{array}{l}B \\ A_{t}\end{array}\right](S)\right\}$ defines an imbedding operator.

The above kind of summation does not produce an imbedding operator when applied more generally, due to failure of the idempotent requirement. The writers have found the "bracket operators" quite useful in constructing examples and counter-examples.

It would appear from the foregoing that $g(P)$ might be very large compared to $P$. Of course, this depends on the structure of $P$ itself, but it is of some interest that for the set $P$ diagrammed below, there are over 1000 distinct imbedding operators. 


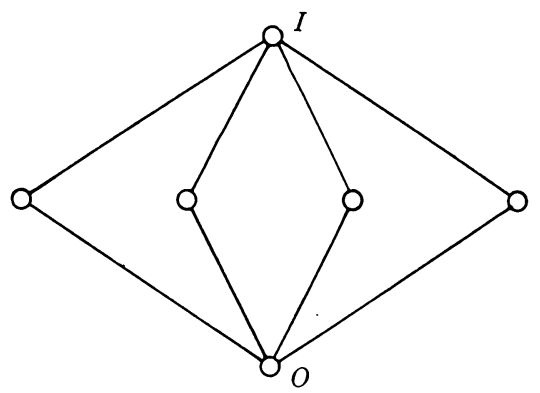

3. Structure of $g(P)$.

Definition. In any partially ordered set, $y$ covers $x$ if and only if (1) $y>x,(2)$ for any $z, y \geqq z>x$ implies $y=z$. If $\phi$ and $\psi$ are imbedding operators and $\psi$ covers $\phi$, we say that $\psi$ is a minimal extension of $\phi$.

Definition. $K$ is a completely irreducible $\phi$-ideal if and only if $K$ is a $\phi$-ideal and is not the intersection of a collection of $\phi$-ideals each different from $K$.

We denote by $\Re(\phi)$ the collection of completely irreducible nonprincipal $\phi$-ideals. If $K \in \mathcal{K}(\phi)$, then $\Sigma(\phi)-\{K\}$ satisfies the definition of a generating collection. Let $\phi_{K}$ denote the imbedding operator generated by $\Sigma(\phi)-\{K\}$, for $K \in \mathcal{K}(\phi)$.

THEOREM 2. For any $\phi \in \mathscr{I}(P), \psi$ covers $\phi$ if and only if $\psi=\phi_{K}$ for some $K \in \mathcal{K}(\phi)$.

Proof. That $\phi_{K}$ covers $\phi$ follows immediately from Lemma D. Assume $\psi$ covers $\phi$. Then by Lemma D, $\Sigma(\phi) \supset \Sigma(\psi)$. Take $K \in \Sigma(\phi)$ $-\Sigma(\psi) . K$ cannot be a principal ideal. Assume $K$ is not in $\Re(\phi)$; then it is the intersection of a collection $\left\{L_{t}\right\} \subseteq \Sigma(\phi)$, each different from $K$. Since $\Sigma(\psi)$ is closed under intersection, there is at least one $L \supset K$ in $\Sigma(\phi)-\Sigma(\psi)$. Consider the set $\Sigma=\Sigma(\psi) \cup\{A \mid A \in \Sigma(\phi), A \subseteq K\}$. This set is a generating collection, and since $K \subset L \in \Sigma(\phi)-\Sigma$, we have $\Sigma(\psi) \subset \Sigma \subset \Sigma(\phi)$. Hence, by Lemma D, $\phi<\phi_{\Sigma}<\psi$, which contradicts our prime assumption. Therefore $K \in \mathfrak{K}(\phi)$ for any $K \in \Sigma(\phi)$ $-\Sigma(\psi)$. But then $\phi<\phi_{K} \leqq \psi$, and so $\phi_{K}=\psi$.

As a corollary, we obtain another characterization of the elements of $\mathcal{K}(\phi)$.

CoROllaRY. $K \in \Re(\phi)$ if and only if there is a minimal extension $\psi$ of $\phi$, with $K \neq \psi(K)$.

EXAMPLE. We give an example of an operator possessing no minimal extension. Let $P$ consist of the elements $O$ and $I$, and an infinite set of pairwise incomparable elements. Define an operator $\phi$ by: 


$$
\phi(S)=\left\{\begin{array}{cl}
\omega(S) & (\text { if } S \text { is finite) } \\
P & \text { (if } S \text { is infinite) }
\end{array}\right.
$$

$\phi$ is an imbedding operator, but the set $\mathfrak{K}(\phi)$ is empty.

Definition. An imbedding operator is inductive if and only if for any chain $\left\{Z_{t}\right\} \subseteq \Sigma(\phi), U\left\{Z_{t}\right\} \in \Sigma(\phi)$.

LEMma F. If $\phi$ is inductive, then for any $S \in \Sigma(\phi)$ and any $x \notin S$, there is a $K \in \Sigma(\phi)$ such that $K \supseteq S$, and $K$ is maximal in $\Sigma(\phi)$ with respect to not containing $x$.

Proof. Apply Zorn's Lemma to the partially ordered set of $\phi$-ideals containing $S$ and not containing $x$.

Lemma G. For any $M$ in $\Sigma(\phi)$, a necessary and sufficient condition that $M$ be completely irreducible is that there exist an $x_{0}$ in $P$ such that $M$ is maximal in $\Sigma(\phi)$ with respect to not containing $x_{0}$.

Proof. It is obvious that the condition is sufficient. Suppose the condition is false. Let $Q=\cap\{K \in \Sigma(\phi) \mid M \subset K\}$. Clearly, $M \subseteq Q$. Suppose $x \notin M$. Then by our assumption, there is a $K_{x}$ in $\Sigma(\phi)$, such that $M \subset K_{x}$ and $x \notin K_{x}$. Hence $x \notin Q$, and so $Q \subseteq M$. But then $Q=M$, and $M$ is therefore not completely irreducible.

Definition. $B_{\psi, \phi}=\{x \in P \mid x \in \psi(S)-\phi(S)$ for some $S \subseteq P\}$.

Lemma $H$. If $K$ is a nonprincipal $\phi$-ideal maximal in $\Sigma(\phi)$ with respect to not containing $x$, then $x \in B_{v, \phi}$. Conversely, if $\phi$ is inductive, and $x \in B_{\nu, \phi}$, then there exists a nonprincipal $\phi$-ideal maximal in $\Sigma(\phi)$ with respect to not containing $x$.

Proof. Let $K$ be maximal in $\Sigma(\phi)$ with respect to not containing $x$, and let $m$ be an arbitrary element of $K^{*}$. Then $m \in K$, since $K$ is nonprincipal. But $\omega(m)=\phi(m) \supset K$, so $x \in \omega(m)$. Since $m$ is arbitrary, $x \in K^{*+}=\nu(K)$. Hence $x \in B_{\nu, \phi}$. Conversely, let $\phi$ be inductive, and let $x \in \nu(S)-\phi(S)$ for some $S \subseteq P$. By Lemma $\mathrm{F}$ there is a $K$ maximal in $\Sigma(\phi)$ with respect to not containing $x$, such that $\phi(S) \subseteq K$. But $x \in \nu(S)=\nu \phi(S) \subseteq \nu \phi(K)=\nu(K)$. If $K$ were a principal ideal, then we would have $\nu(K)=K$, and $x \in K$. Hence $K$ is nonprincipal.

Theorem 3. If $\phi$ is inductive and $\psi>\phi$, then there exists a $K \in \mathcal{K}(\phi)$ such that $\psi \geqq \phi_{K}$.

Proof. Since $\psi>\phi$, there exists $A \in \Sigma(\phi), \psi(A) \supset A$. Take $x \in \psi(A)$ $-A$. Then $x \in B_{\boldsymbol{\nu}, \phi}$, and by Lemmas $\mathrm{G}$ and $\mathrm{H}$ there is a $K \in \mathcal{K}(\phi)$ such that $K \supseteq A$ and $x \notin K$. But $x \in \psi(A) \subseteq \psi(K)$. Hence $K \notin \Sigma(\psi)$, and so $\Sigma(\psi) \subseteq \Sigma(\phi)-\{K\}$. Therefore $\psi \geqq \phi_{K}$. 
Definitions. An element $x$ in a partially ordered set $P$ is an atom if and only if $x$ covers $0 . P$ is atomic if and only if for any element $y \neq 0$, there is an atom $x$ such that $y \geqq x$. Theorems 2 and 3 state that if $\phi$ is inductive, then $\{\psi \mid \psi \geqq \phi\}$ is an atomic sublattice of $g(P)$. We also have the following result:

Theorem 4. For any $\phi$ in $g(P)$, the lattice $\{\psi \mid \psi \geqq \phi\}$ contains a sublattice isomorphic to the Boolean algebra of all subsets of its atoms.

Proof. By Theorem 2 the atoms of $\{\psi \mid \psi \geqq \phi\}$ are in one-to-one correspondence with the elements of $\mathscr{K}(\phi)$. The set of generating collections of form $\Sigma(\phi)-\mathscr{L}$, for $\mathscr{L} \subseteq \mathcal{K}(\phi)$, when partially ordered by inclusion, is anti-isomorphic to the Boolean algebra $\mathfrak{A}$ of all subsets of $\mathfrak{K}(\phi)$. Hence the set of corresponding operators is isomorphic to $\mathfrak{A}$ by Lemma $\mathrm{D}$, and so to the Boolean algebra of all subsets of the atoms of $\{\psi \mid \psi \geqq \phi\}$.

COROLlaRY $1 . \mathscr{g}(P)$ is atomic and contains a sublattice isomorphic to the lattice of subsets of its atoms.

Proof. Lemma $B$ states that $\omega$ is additive and hence inductive.

REMARK. $\mathscr{K}(\omega)$ is in 1:1 correspondence with $B_{\nu, \omega}$. For if $x \in B_{\nu, \omega}$, the set $P-\{x\}^{*}$ is an $\omega$-ideal, maximal in $\Sigma(\omega)$ with respect to not containing $x$, and clearly the only such maximal ideal. Thus the atoms of $\mathfrak{g}(P)$ are also in $1: 1$ correspondence with $B_{\nu, \omega}$. This leads to:

Corollary 2. If $\mathscr{g}(P)$ is a chain, then $B_{v, \omega}$ contains at most one element.

Following Birkhoff [1], we call $P \uparrow$-atomic if and only if $x<y$ always implies that there is a maximal chain connecting $x$ and $y$ which is well-ordered. Clearly $\uparrow$-atomic implies atomic. By a standard transfinite argument, we get:

COROllary 3. If each $\phi$ in $\mathscr{g}(P)$ different from $\nu$ is inductive, then $\mathfrak{I}(P)$ is $\uparrow$-atomic.

A result related to Theorem 4 is the following:

THEOREM 5. If

(1) $P$ is a chain,

(2) $G=\{x \in P \mid x$ is the l.u.b. of an infinite ascending chain in $P\}$, then $\mathscr{g}(P)$ is isomorphic to $2^{G}$.

Proof. It is clear that in this case, $B_{\nu, \omega}=G$, and each $K \in \mathscr{K}(\omega)$ is of the form: $K(a)=\{x \in P \mid x<a\}$ for some $a \in G$. Thus the atoms of $\mathfrak{I}(P)$ are in $1: 1$ correspondence with $G$. Since $\mathscr{g}(P)$ is a complete lattice, each set of atoms of $g(P)$ has a unique l.u.b. Therefore we 
need only show that each $\phi$ in $g(P)$ is the l.u.b. of a unique set of atoms. With each $\phi$ in $\mathscr{g}(P)$ we associate the subset $B_{\phi, \omega} \subseteq G$. Then $\Sigma(\phi)=\Sigma(\omega)-\left\{K(a) \mid a \in B_{\phi, \omega}\right\}=\bigcap\left\{\Sigma\left(\phi_{K(a)}\right) \mid a \in B_{\phi, \omega}\right\}$. But by Lemma $\mathrm{D}$, this means that $\phi=\mathrm{V}\left\{\phi_{K(a)} \mid a \in B_{\phi, \omega}\right\}$. This representation of $\phi$ is clearly unique.

4. A sufficient condition for $g(P)$ to be a chain. We first give an example to show that the converse of Corollary 2 to Theorem 4 is false. In the adjoining figure, the criss-cross structure of $x_{i}$ 's and $y_{i}$ 's continues upward without limit, and is surmounted by an infinite discrete chain descending from $I$. The only element of $B_{\nu, \omega}$ is $p$, but the operators $\left.x_{2}, y_{1}\right]$ and $\left.x_{1}, y_{2}\right]$ are incomparable.

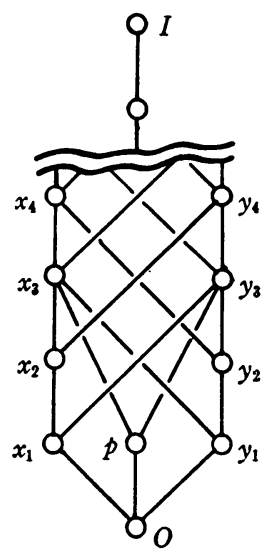

With some slight additional hypothesis on $P$, we shall now prove a converse to the above Corollary 2. This hypothesis is not necessary, as will be shown in the next figure.

Definition. $P$ has the minimal condition if and only if for any subset $A$ of $P, A^{*}$ has a minimal element.

TheOREM 6. If (i) $P$ is a lattice, or $P$ has the minimal condition, and (ii) $B_{v, \omega}$ contains at most one element, then $g(P)$ is a chain.

Proof. Assume that $g(P)$ is not a chain, and that $B_{r, \omega}=\{p\}$. Then there exist operators $\phi_{1}$ and $\phi_{2}$, and subsets $A$ and $B$ (which we may take as $\omega$-closed) such that:

$$
\begin{array}{ll}
\phi_{1}(A)=A, & \phi_{1}(B)=B \cup\{p\}=\nu(B), \\
\phi_{2}(A)=A \cup\{p\}=\nu(A), & \phi_{2}(B)=B
\end{array}
$$

and such that $p \notin A, p \in B$. 
Assertion 1. $A$ and $B$ are incomparable sets. For if $A \subseteq B$, then $\phi_{2}(A) \subseteq \phi_{2}(B)$, and so $p \in B$.

Assertion 2. There exist $a \in A$, and $b \in B$, such that $a, b$, and $p$ are pairwise incomparable.

Proof of 2. If $A-B \subseteq \omega(p)$, then $\phi_{1}(B)=B \cup\{p\}=B \cup \omega(p)$ $\supseteq A-B$. This means that $A \subseteq B \cup\{p\}$; i.e., since $p \notin A, A \subseteq B$. But this contradicts Assertion 1, and therefore there exists an $a \in A-B$, $a \vdots p$. Likewise there exists a $b \in B-A, b \leq p$. Since $A$ and $B$ are $\omega$-closed, $a$ and $b$ are incomparable, and also $p \nless a$ and $p \nless b$. Thus $p, a$ and $p, b$ are also incomparable pairs.

Now if $P$ is a lattice, $\nu(\{a, b\})=\omega(a \bigvee b)$. Hence $a \bigvee b \in \nu(\{a, b\})$ $-\omega(\{a, b\})$, and $a \bigvee b \neq p$, a contradiction of the prime assumption.

Assume now that $P$ has the minimal condition. Let $\Delta(P)$ be the set of all pairs of incomparable elements $\{x, y\}$ contained in $\{a, b\}^{*}$. $\Delta(P)$ can be partially ordered by:

$$
\{x, y\}<\{u, v\} \text { if and only if }\{u, v\} \subseteq\{x, y\}^{*} .
$$

Now let $\Omega$ be a maximal chain in $\Delta(P)$. If $Q=U\{\{x, y\} \mid\{x, y\} \in \Omega\}$ then $Q^{*}$ contains no pair of incomparable elements; i.e., $Q^{*}$ is a chain in $P$. It has a least element $m$ by the minimal condition, and so $m \in \nu(Q)-\omega(Q)$. But $Q^{*} \subseteq\{a, b\}^{*}$, whence $m \neq p$, a contradiction.

As a final result, we obtain a condition on $P$ which, if $g(P)$ is a chain, implies that $\mathscr{I}(P)=\{\omega, \nu\}$, at most. To this end, let $C$ denote the maximal chain in any partially ordered set $P$, which satisfies the two conditions: (i) $I \in C$, and (ii) $x \in C$ implies $x \in C^{+}$. The existence of such a maximal chain is assured by Zorn's Lemma, and it is obviously unique.

Theorem 7. Let $P$ be a partially ordered set in which $C$ contains a least element. Then if $\mathfrak{g}(P)$ is a chain, it contains at most the operators $\omega$ and $\nu$.

Proof. If $B_{v, \omega}$ is vacuous, then $g(P)$ contains only the operator $\omega$. So let us suppose that $B_{\nu, \omega}=\{p\}$, in accord with Corollary 2 of Theorem 4. Let $m$ be the least element of the chain $C$, and let $K$ $=\{x \in P \mid x<m\} . K$ and $K^{*}$ have no element in common, for if they did, it could be adjoined to the chain $C$, which would contradict the maximality of $C$. Hence $m$ is the least element of $K^{*}$, and $K^{*+}=\nu(K)$ $=\omega(m)$. Hence $m \in \nu(K)-\omega(K)$ and thus $m=p$. Note that $K$ is a completely irreducible, nonprincipal $\omega$-ideal. We now show that $\Sigma(\omega)-\{K\}=\Sigma(\nu)$. Suppose the contrary; then there exists an $\omega-$ closed set $A \subset P$, with $\nu(A)-A=\{p\}$ and $A \neq K$. Since $p=m \notin A$ 
we must have $A \subset K$. Suppose $q \in K-A$. Since $\nu(A)=\omega(p)$, it follows that $q \in \nu(A)-A \subseteq B_{\nu, \omega}$. But $q \neq p$, a contradiction. Hence the operator $\nu$ covers $\omega$, proving the theorem.

The following figures illustrate: (1) the added condition (i) in the hypothesis of Theorem 6 is not necessary, (2) one can construct a partially ordered set $P$ such that $g(P)$ is a chain of any given finite length, (3) the hypothesis of Theorem 7 is not a necessary condition.
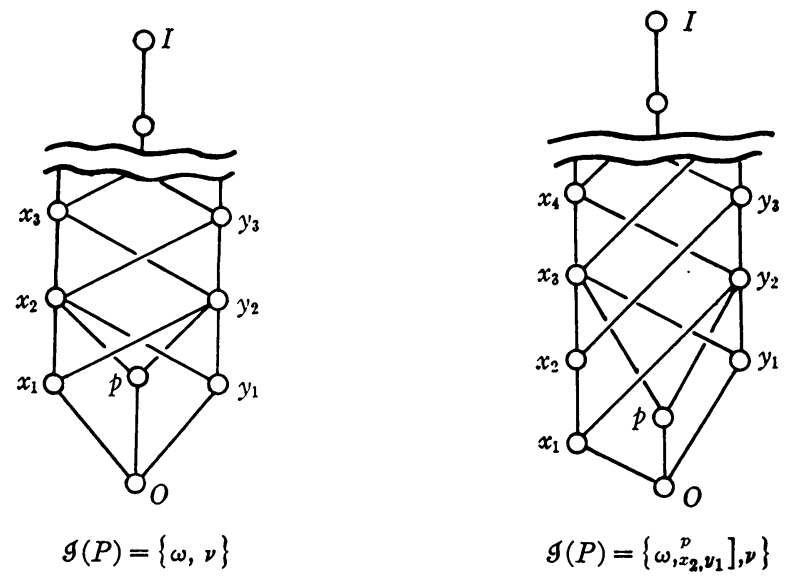

$$
\mathscr{g}(P)=\{\omega, \nu\}
$$

\section{REFERENCES} 1948

1. G. Birkhoff, Lattice theory, Amer. Math. Soc. Colloquium Publications, vol. 25,

2. R. P. Dilworth and J. E. McLaughlin, Distributivity in lattices, Duke Math. J. vol. 19 (1952) pp. 683-693.

3. M. Ward, The closure operators of a lattice, Ann. of Math. vol. 43 (1942) pp. 191-196.

UNIVERSITY OF CONNECTICUT 